\title{
Gross and Histopathological Lesions Associated with Escherichia coli Infection in Chickens Examined, At Ecwa Veterinary Clinic, Bukuru, Plateau State, Nigeria
}

\author{
Barde Israel Joshua ${ }^{1 *}$, Lar Richard Nanchak ${ }^{2}$, Agbi Christiana Ayo², \\ Barde Dorcas Jummai ${ }^{3}$, Logyang Lot Emmanuel ${ }^{1}$, Isa Suleiman', Umaru \\ Mada Alesa ${ }^{1}$, Ugbe Aondona Dominic ${ }^{1}$, Choji Tobias Peter Pwajok ${ }^{1}$, \\ Dashe Yakubu Gunya ${ }^{1}$ and Ishaku Leo Elisha ${ }^{1}$ \\ ${ }^{1}$ National Veterinary Research Institute, Vom, Plateau State, Nigeria \\ ${ }^{2}$ Federal College of Animal Health and Production Technology, Vom, Plateau \\ State, Nigeria
}

${ }^{3}$ Nasarawa State University Keffi, Nigeria

*Corresponding Author: Barde Israel Joshua, National Veterinary Research Institute, Vom, Plateau State, Nigeria.
Received: January 04, 2022

Published: February 25, 2022

(C) All rights are reserved by Barde Israel Joshua., et al.

\begin{abstract}
Colibacillosis is a common bacterial disease of economic importance in poultry through decreasing the infected birds' productivity, increase mortality, condemnation of infected carcasses at slaughter and prophylactic and treatment cost and is report worldwide. Information on gross lesion and histopathological associated with natural Colibacillosis in chicken in Nigeria is scanty, hence the need for this study. The outcome of the study may become useful information on the gross and histopathological lesions of importance to students and as well to poultry diagnosticians on the risk and possible threat posed by E. coli infection in chicken. The study was conducted at ECWA Veterinary Clinic Bukuru in Jos South Local Government Area of Plateau State, Nigeria. The sample comprise of mortalities brought to ECWA Veterinary Clinic for postmortem examination. The suspected cases of colibacillosis in the chicken were identified based on the clinical signs and gross lesions after following a though postmortem examination of birds and laboratory isolation of Escherichia coli. The gross and histopathology lesion associated with colibacillosis as observed from various chickens, showed as follows: thick fibrin layer attached to the liver, Fibrinious deposit on the air sac (air saculitis), necrotic foci on kidney, enlargement and congestion of spleen, thick fibrin layer attached to heart (pericarditis), thick fibrin layer attached to liver (perihepatitis), enlargement of the spleen (splenomegaly), broken egg in the abdominal cavity (egg yolk peritonitis and salpingitis). The histopathological lesions associated with colibacillosis revealed multifocal necrosis along with depletion of the lymphoid cells, glomerulonephritis, the intestinal villi showed a sloughing and necrotic villi, necrotic hepatocytes, and necrosis of the renal tubules. Evidence from the findings of the study shows that colibacillosis exist around Bukuru, Jos South Local Government Area of Plateau State. In view of the above, Battery cage system is recommended to reduce feed and water contamination with faecal material and strict bio security is advocated.
\end{abstract}

Keywords: Avian Colibacillosis; Diagnosis; Bukuru; Nigeria

\section{Introduction}

The infection caused by the extra internal pathogenic Escherichia coli (EXPEC) is termed colibacilliosis which is an infectious disease characterized by acute fatal septicemia or sub fibrinous pericardititis, air sacculitis, salpingitis, and peritonitis affecting chickens of various ages. Colibacillosis is a common bacterial dis- ease of economic importance in poultry through decreasing the infected birds' productivity, increase mortality, condemnation of infected carcasses at slaughter and prophylactic and treatment cost and is report worldwide [1]. Signs are nonspecific and vary with age, organs involved and concurrent disease. Young bird dying of acute septicemia have few lesions such as enlarged hyperemic 
Gross and Histopathological Lesions Associated with Escherichia coli Infection in Chickens Examined, At Ecwa Veterinary Clinic, Bukuru, Plateau State, Nigeria

liver and spleen with increased fluid in body cavities other lesions include, pneumonia, arthritis, and osteomyelitis [1]. Avian colibacillosis is a major infectious disease in bird of all ages, which impart a significant economic impact on poultry production. Loses are economical, as a result of mortality and decrease productivity of affected birds. Mainly around the peak egg production period and throughout the late lay period [2]. Gross lesion is one that can be seen with a naked eye, gross lesion is chiefly achieve by a process, known as necropsy (synonym: postmortem examination) which is the systematic examination of a carcass with a view to searching for lesions that may point to animal's death, it is a very important diagnostic tool that is used to support other procedure performed in the diagnoses of disease conditions of the animal, birds and fish/ shell fish [3]. There are several reason for performing postmortem examination. These include; Finding the cause of death, Confirming a diagnosis, investigation unsuccessful therapy, increasing knowledge or satisfy curiosity. The pathologist uses the gross description, culture result and other data as well as the cytological and histology appearance of the lesion to make a diagnosis [4].

Diagnostic based on gross examination can be made accurately on up to $90 \%$ of samples. This is the importance of gross description. However, for macroscopic pathology to be of help in establishing a diagnosis; knowledge, precision and elegance in gross description are required [5].

Escherichia coli, a gram-negative rod shape facultative anaerobic bacteria belong to the family Enterobacteriacea. E. coli is a natural inhabitant of gastrointestinal tract both producing disease under certain stressful conditions such as overcrowding, poor ventilation, malnutrition, extreme temperature and immune suppression [6]; it is one of the main cause of economic losses in the poultry industry $[7,8]$, particularly in broilers chickens through mobility, lack of flock growth uniformity, lowered production, increased condemnation at slaughter plants and mortality [9]. However E. coli infection in poultry is associated with verities of disease condition in poultry ranging from pericarditis, omphalitist, celulitis, coli septicemia, coligranuloma to swollen head syndrome [10].

Any localized or systemic infection characterized by edisepticemic, hemorrhagic coliform coligranuloma (Hjarres disease), swollen head syndrome, venereal colibaccillosis, coliform cellulitis, peritonitis, orchitis, osteomyelitis/synovitis, omphalitis/yolk sack infection and enteritis, referred to as colibacillosis. The acute form of the disease is characterized by scepticemia resulting to death, and its supacute formed by peritoneal, perihepatitis, airsaculitis and other lesion.

The presence of exudation in the peritoneal (abdominal) cavity including serum fibrin and inflammatory cells (Pus), are the characteristic. Fibrin is the product of inflammatory response in chicken that is seen covering the surface of multiple organs, including the oviduct, ovary, the intestine, the alveoli, the heart, the lungs and the liver [2]. However, we should not only diagnose base on the lesions to infer an infection with Escherichia coli without adding the descriptor "Coli" or "Coliform", because other opportunistic bacteria can induce similar to E. coli infection [11]. Information on gross lesion and histopathological associated with natural Colibacillosis in chicken in Nigeria is scanty, hence the need for this study. Relatively few studies have mentioned gross and histopathological lesions associated with natural colibacillosis in chicken, hence the need to carry out this study. The outcome of the study may become useful information on the gross lesions of importance to the student and as well to the surrounding community on the risk and possible threat posed by $E$. coli infection in chicken.

The analysis will also aid in the identification of the organs involved with gross lesion that are associated with $E$. coli infection in chicken which will effectively help the pathologist. The study will go a long way in tackling the cases associated with $E$. coli infection.

\section{Materials and Methods}

Study area

The study was conducted at ECWA Veterinary Clinic Bukuru in Jos South Local Government Area of Plateau State, Nigeria. Jos South with it headquarter at Bukuru $9^{\circ} 4800^{\prime \prime} \mathrm{Nx} 8^{\circ} 5200^{\prime \prime} \mathrm{E}$. it has an area of $510 \mathrm{~m} 2$ and the population of 306,716 as at the 2006 census.

\section{Sample collection}

The sample comprised of mortalities which were brought at ECWA Veterinary Laboratory Clinic for postmortem examination. The suspected cases of colibacillosis in the chicken were identified based on the history clinical sign and lesions after following a 
Gross and Histopathological Lesions Associated with Escherichia coli Infection in Chickens Examined, At Ecwa Veterinary Clinic, Bukuru, Plateau State, Nigeria

though postmortem examination of birds. History of each suspected flocked were flock size, mortality and total number of the flock.

Representation sample (heart, liver, intestine, kidney and spleen).

\section{Sample preparation}

- The samples of organs that show gross lesion were photographed and label accordingly from colibacillosis suspected chickens. The tissues were fixed in $10 \%$ formalin

- $\quad$ The fixed samples were transported and submitted to histhopatological laboratory units of central Diagnostic laboratory of National Veterinary Research Institutes Vom Plateau State, for histhopatological preparation according to the standard operation procedure in histhopatology [12].

\section{Necropsy procedure}

\section{Examination of the carcass}

The assessment of the general appearance of the feather, weight, pigmentation of skin and shanks, physical injuries, facial tissue, eye, feces, nasal and respiratory discharge, leg join deformities and external parasite.

\section{Opening of the Body cavity}

The carcass is grasped by both legs, which are pulled laterally to break the skin over the breast and the same time losing the coxofemoral joints. It enable the bind to lie flat on it back and then the skin will be cut which serve as the first point of incision. With the aid of a knife a straight line incision is made through the abdomen and forward along the rib articulations, and then the breast can be removed, exposing both the abdominal and pleural cavities. Observed the presence of fluids, exudate, or blood in either cavity. The visceral contents are examined in situ for any gross lesion before they are removed if any gross lesion it's been photographed.

\section{Examination of the organs}

This begins by cutting gastrointestinal tract at the termination of the proventriculus, gizzard, the pancreas which rest on duodenal loop, and the large intestine include ceca are removed. The liver and the spleen are removed together, any lesion in the liver and the enlargement of the spleen is been photographed. The lobulated kidney which is closely attached with bony structure of the sub lumber region is observed if any changes in the kidney it's been photographed. The oviduct is removed and examine in situ, for any exudate which can be seen externally as a discharge e.g. Egg peritonitis and salpingitis. The heart is examined in situ for any fibrin exudate if any is a sign of pericarditis it's been photographed. The air sac is examine which is present in the both the thorax and abdomen, are form by a thin layer membrane normally unnoticed unless is fill with exudate. When it appears cloudy covers an accumulation of whitish yellow exudate. Is term air sacculitis.

\section{Histopathological techniques}

- $\quad$ Fixation: The piece of tissue preferably $3-4 \mathrm{~mm}$ thick is fixed in the common routine fixative ( $10 \%$ formalin) for 24 hours. The purpose generally is to preserve the tissue in a life-like manner and permit subsequent treatment of the specimen with other processes.

- Dehydration: This is a process in which water contained in the tissue is removed using ascending grades of alcohol, starting from 70\% through three batches of absolute alcohol. 70\% alcohol for 60 minutes $90 \%$ alcohol 45 minutes, absolute alcohol for 45 minutes.

- Clearing: The alcohol is removed from the dehydrated tissue. This is necessary for impregnation to take place, since alcohol, like water dose not mixed with paraffin wax. The chemical use to remove the alcohol is called clearing agent known as xylene. The tissue is placed in three phase of xylene each for 60 minutes.

- Impregnation: Here the tissue is infiltrated with molten paraffin wax, the purpose of which is to make the tissue suitable for microtomy. Infiltration of paraffin wax, at 30 minutes, at 60 minutes and at 90 minutes.

- Blocking out: After impregnation with molten paraffin wax, the tissue begins embedded in molten paraffin wax in a mould meant for embedding. This is done by filling the mould with molten paraffin wax and the tissue is placed on the Centre bottom of the mould. After solidification and the mould is immersed in cool water for easy removal of the paraffin block.

- $\quad$ Trimming: The block is moved forward so that the wax block is almost touching the knife. To trim away surplus wax and to expose a suitable area of the tissue for sectioning, the section thickness is adjusted at 15 microns. An ice is then applies to the surface of the block for few second and wipe surface of block free of water. 
The microtome is now moved in an easy rhythm with right hand operating a microtome and left hand holding the section away from the knife. Then ribbon is formed due to the sight heat generated during cutting which causes the edge of the section to adhere.

\section{Floating}

The cut paraffin embedded tissue is placed in the thermostatically controlled water at a temperature of about $4-6 c^{\circ}$ below melting point of paraffin wax. The section is then flouted flat and the ribbon as come to rest on water. The remaining wrinkles and fold are removed by tiring apart with a forceps.

\section{Picking up section}

The process of picking up a section on a slide is achieved by immersing the slide lightly, smeared with adhesive vertical to fourth of its length bringing the section in contact with the slide.

On lifting the slide vertically from the water the section will flatting on the slide. The sections are then blottened by air dry it on the staining rack.

\section{Staining techniques}

- It is stain with haematoxylin/eosin.

- The section were dewax in two changes of xylene and hydrated in water through series of alcohol of descending order of concentration of $90,80,70$ each for 10 minutes.

- $\quad$ The slide is then stained in haematoxylin for 5 minutes.

\section{Rinsed in tap water}

- $\quad$ Treated in scot's water substituted for 5 minutes (to deepen the blue colour of the nucle i). Washed in tap water and counterstained in $1 \%$ aqueous eosin for 2 minutes and wash. Dehydrated in series of alcohol in ascending order of concentration (70\%, 80\%, 90\% absolute I and ii). 10 minutes for each.

- $\quad$ Then section were finally cleared and mounted with cover slip using Canada blalsum (D P X) dibutylphthalute polystyrene xylene.

- $\quad$ Then viewing the slide [12].

\section{Results}

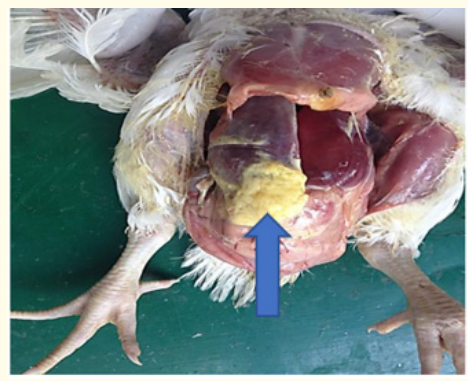

Figure 1: Broiler chicken affected with severe colibacillosis showing thick fibrin layer attached to liver (arrow).

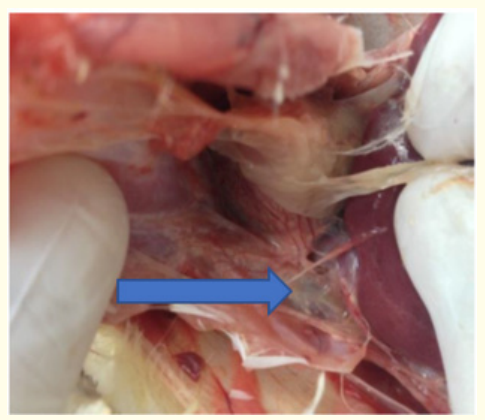

Figure 2: Chicken affected with colibacillosis showing fibrinous deposit on thoracic air sacs (arrow).

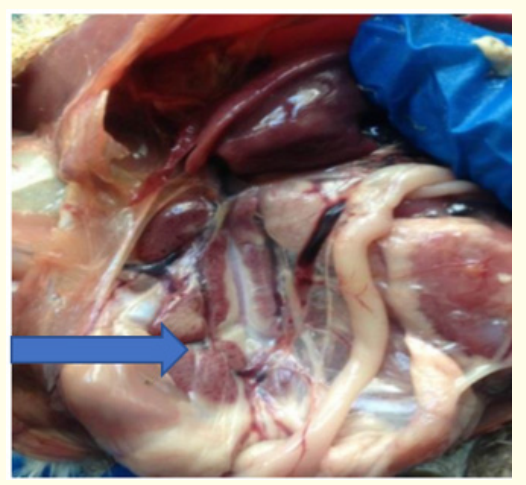

Figure 3: Colibacillosis affected broiler chicken revealing necrotic foci on kidneys (arrow). 


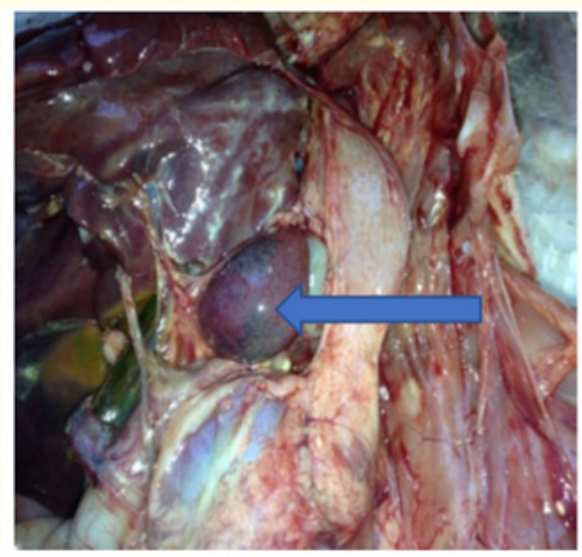

Figure 4: Broiler chicken affected with colibacillosis revealing enlargement and congestion of spleen (arrow).

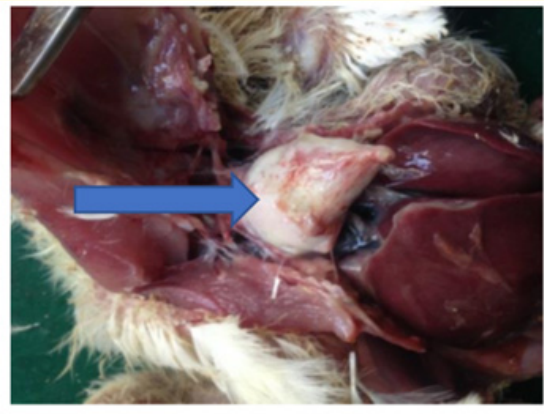

Figure 5: Broiler chicken affected with severe colibacillosis showing thick fibrin layer attached to heart (arrow).

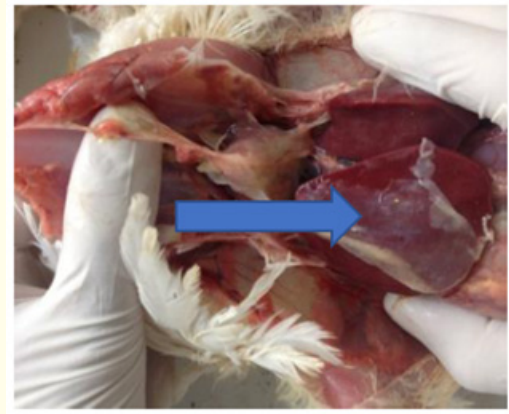

Figure 6: Broiler chicken affected with colibacillosis showing thin fibrin layer attached to liver (arrow).

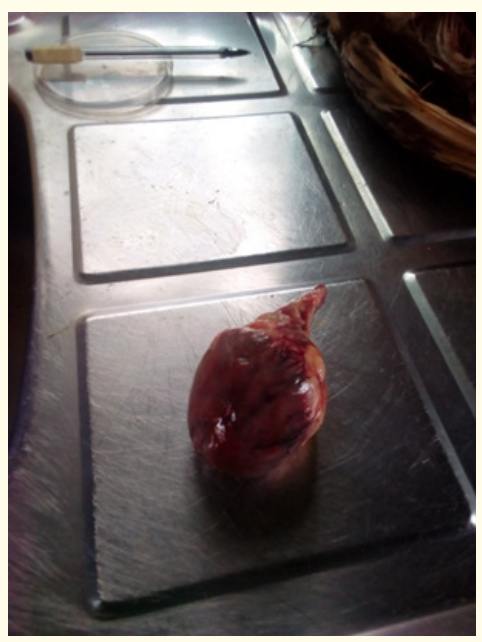

Figure 7: Layer chicken affected with colibacillosis showing enlarged spleen

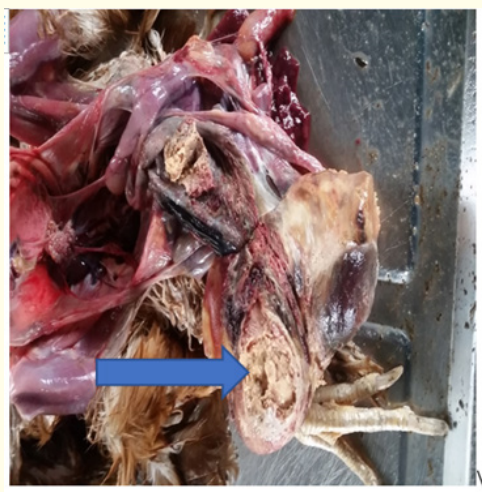

Figure 8: Layer chicken affected with colibacillosis showing inflammation of the peritoneum (arrow).

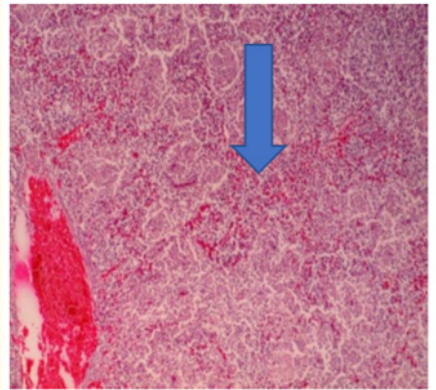

Figure 9: Photomicrograph of the spleen from colibacillosis affected chicken revealing multifocal necrosis along with depletion of lymphoid cells. H.E. $\times 100$. 


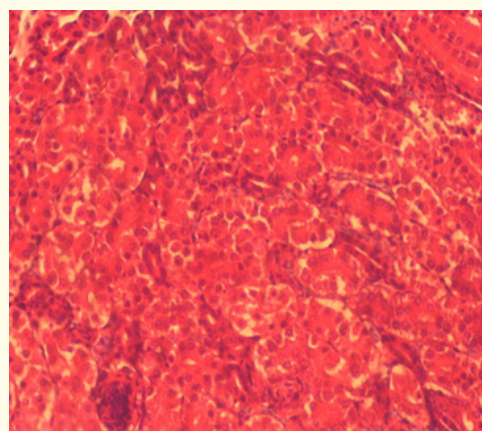

Figure 10: Photomicrograph of the kidney Showing necrotic glomerulus and renal tubules. H.E x 400.

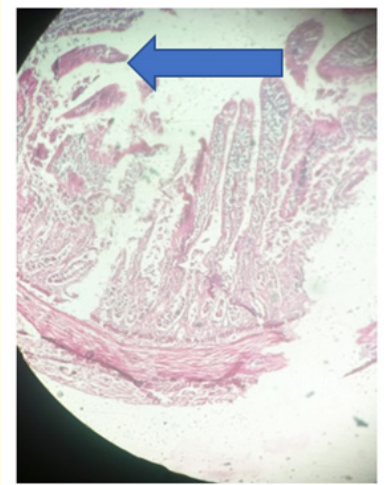

Figure 11: Photomicrograph of the intestinal Mucosa showing sloughing and degenerated Villi and Ilium. X400.

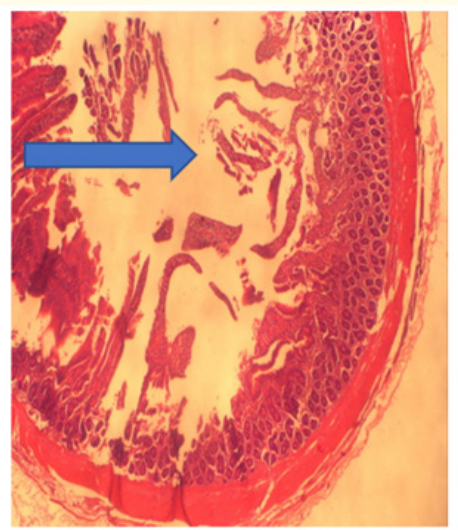

Figure 12: Photomicrograph of the intestinal. mucosa showing degenerated and necrotic villi $\mathrm{x} 400$.

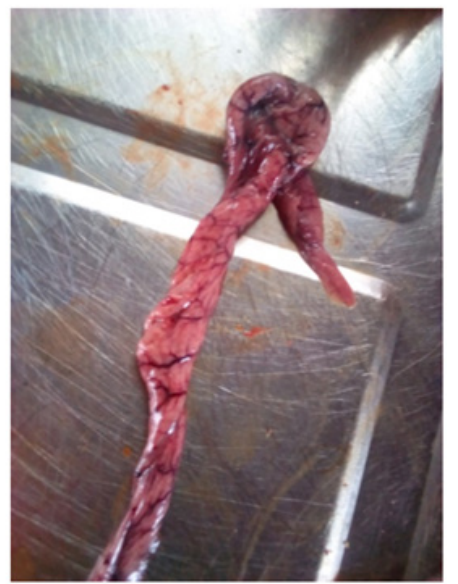

Figure 13: Acute salpingitis from layer chicken.

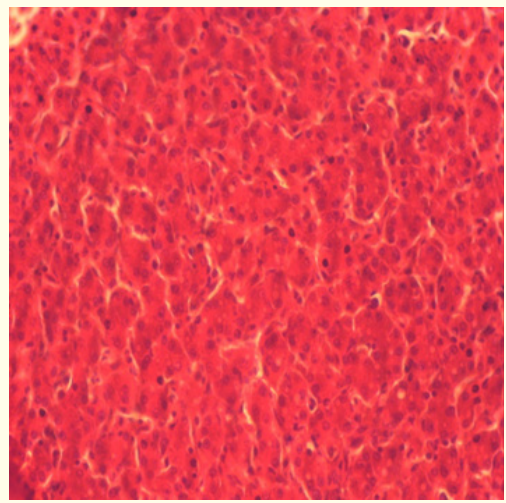

Figure 14: Necrotic hepatocyte k x400.

\section{Discussion}

Years ago, both incidence and severity of colibacillosis have rapidly increased and current scenario alarms that is likely to grasp its hold in future and thus impose a great threat to poultry industry [13]. The infection caused by the EXPEC is termed coli bacilliosis which is an infectious disease characterized by acute fatal septicemia or sub fibrinous pericardititis, air sacculitis, salpingitis, and peritonitis affecting broiler chickens aged 4-6 weeks. Colibacillosis is a common bacterial disease of economic importance in poultry through decreasing the infected birds' productivity increase mortality, condemnation of infected carcasses at slaughter and prophylactis and treatment cost and is report worldwide. 
Gross and Histopathological Lesions Associated with Escherichia coli Infection in Chickens Examined, At Ecwa Veterinary Clinic, Bukuru, Plateau State, Nigeria

Figures 1, 2, 5 and 6 showed fibrin deposit on the liver, heart and thoraxic airsacs respectively (fibrinous hepatitis, pericarditis and air sacculitis) which are all indicative of acute condition. Figure 3 shows necrotic nephritis while figures 4 and 7 showed splenomegaly. Figure 9 shows necrotic splenitis while figures 10 and 15 showed necrotic glomerulonephritis. Figures 11, 12 and 13 showed necrotic enteritis while figure 16 showed salpingitis. These findings are in agreement with reports of previous researchers [14], who reported air sacculitis, and Nagi and Khana, [15] who reported similar lesions in liver, kidneys, spleen, heart and intestine.

More so in figure 14 gross pathological lesions were severe in liver which comprise of necrotic hepatocyte, with the nuclei shrunken and disposition of fibrinous exudates of the surface of the liver. The result were similar to the finding of Kumar., et al. [16] who reported disposition of fibrinous exudates on the liver surface beside the liver other changes. Renu., et al. [17] also reported thick fibrous later on all visceral organisms' avian colibacillosis. Grossly heart generally revealed congestion, variable deposition of fibrous layer on pericardium and adhesions of heat with chest cavity. Similarly types of lesions have been described by Nakamlura., et al. [18]. Air sac were generally cloudy and covered with a thin to thick layer of fibrin. Lesions were prominent in thoracic air sac than in abdominal air sac. The result are similar with those of Sylvester., et al. $[19,20]$, who reported air sarculities, from clinical case of different field outbreaks. Figure 4 grossly spleen revealed slight enlargement and variable degrees of congestion and presence of congestion in Figure 7 the spleen of a large chicken revealed enlargement of the spleen. Figure 8 grossly egg Peritonitis which is the inflammation of the Peritoneum caused by the presence of the broken egg in the abdominal cavity. The finding is current with the finding of [21]. Which shows scattered pieces of Yolk, Thick yolk, Cheeselike material or milky fluid in the abdominal cavity when affected with E. coli. Figure 13 grossly show salpingitis from layer chicken, which is the inflammation of oviduct due to colibacillosis.

\section{Conclusion}

Carefully post mortem examination supported by one or more antimortem laboratory investigation can greatly assist in arriving at confirmatory diagnosis. Diagnostic poultry pathology involves a multidisciplinary approach to many poultry diseases ranging from histopathology, clinical pathology, toxicology, microbiology etc.

\section{Recommendation}

Evidence from the findings of the study shows that colibacillosis exist around Bukuru, Jos South Local Government Area of Plateau State. In view of the above, Battery cage system is recommended to reduce feed and water contamination with faecal material and strict bio security is advocated.

\section{Bibliography}

1. Seneviratna P. "Diseases of poultry (including case birds) (2nd edition)". Bristol: John Wright and Sons Ltd (1999): 68-70.

2. Linden O. "Colibacillosis in Layers: an overview". The Poultry site from (2015).

3. Taiwo VO. "A manual for necropsy procedure for veterinary students and Unician Dabfol print and pack limited". Dagbe, Ibadan, Nigeria (2005): 2-21.

4. Latimer AL and Rakich PM. Necropsy examination: Bitchi Bio, Medicine: Medicine: Principle and application wingers publishing Lake Worth (1994): 355-411.

5. Geller SA and Horowitz RE. "Gross Examination". in: Day C.(editions) Histopathology method in molecular Biology (Methods and Protocols), Human Press, New York 1180 (2014): 9-34.

6. Kabir SML. "Avian colibacillosis and Salmonellosis: A closer look at epidemiology, pathogensis, diagnosis, control and public health". International Journal of Environmental Research and Public Heath 7.1 (2010): 89-114.

7. Yogaratnam K. "Analysis of the causes of high rate of carcass rejection at a poultry processing plant". Veterinary Record 137.9 (1995): 217.

8. Ewers C., et al. "Avian Pathogenic Escherichia coli (APEC)". Berlin undMunchener Tierar Ztliche Woehenschrift, 116.9-10 (2003): 381-395.

9. Gross WB. "Colibacillosis". In Disease of poultry". (BW Cainek, editor). Nine edition. Lowa State University Press, Ames (1991): 138-144. 
10. Wray C., et al. "Enterobacteriaceae". In: poultry Disease. (FTW Jordan, M Pattison, editors). WB Sauders, Cambridges, UK (1996): 43.

11. Barnes J., et al. "Colibacilosis Disease of Poultry". Saif YM. Lowa Blackwell Publishing Professional. 12 (2008): 716-762.

12. Paget EG and Thompson R. "Standard operating procedures". In pathology, MTP Press, Lancaster (1979): 134-139.

13. Rodriquez-Siek KE., et al. "Comparison of Escherichia coli. Isolated implication in human curinary tract infection. And avian colibacillosis". Microbiology 5 (2005): 2097-2110.

14. Cheville NF and Arp LH. "Comparative Pathological Findings of Escherichia coli infection in brid". Journal of the American Veterinary Medical Association 173.5 (1978): 584-587.

15. Nagi MS and Khannu PN. "A cholera like disease in chicken to haemogtic Escherichia coli". Indian Journal of Veterinary 44.8 (1967): 629-633.

16. Kumar A., et al. "Pathological studies of natural cases of colibacillosis in Harguna Satate". Haryana Veterinarian 52 (2013): 118-120.

17. Renu LMS., et al. "Pathological studies on poultry mortality with reference to Esherichia coli infections". Indian Journal of Poultry Science 47 (2012): 222-226.

18. Nakamlura K., et al. "Effect of infectious basal disease virus on infections produced by Escherichia coli of high and low virulence in chorines". Avian Pathology 19 (1985): 713-721.

19. Sylvester SN., et al. "In-Ove and in Vivo pathogenicity study of avian Escherichia Coli isolated from cases of colibacillosis in chicken". Indian Journal of Veterinary Pathology 3.1 (2005): 225-230.

20. Gungane GR., et al. "Studies on experimental colibacillosis in chicks”. Indian Veterinary Journal 83 (2006): 118-119.

21. Rosales AG. "Egg peritonitis". S in Poultry (Egg tolk peritonitis) (2019).

\section{Assets from publication with us}

- Prompt Acknowledgement after receiving the article

- Thorough Double blinded peer review

- Rapid Publication

- Issue of Publication Certificate

- High visibility of your Published work

Website: www.actascientific.com/

Submit Article: www.actascientific.com/submission.php Email us: editor@actascientific.com

Contact us: +919182824667 